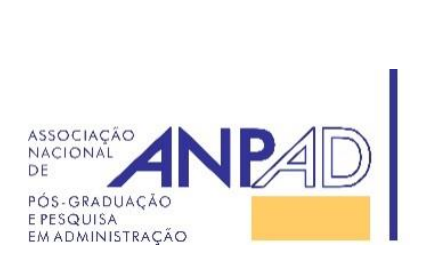
Disponível em
http://www.anpad.org.br/rac
RAC, Rio de Janeiro, v. 21, n. 5, art. 4,
pp. 666-684, Setembro/Outubro, 2017
http://dx.doi.org/10.1590/1982-7849rac2017160189
$(c c)$ EY

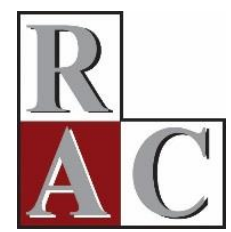

\title{
Socializar para Adequar-se: Como Redes Sociais Intraorganizacionais Podem Influenciar a Adequação Indivíduo- Organização
}

\author{
Socialize to Fit: How Intra-organizational Social Networks Can Influence Person- \\ Organization Fit
}

Maria Gabriela de Cássia Miranda ${ }^{1}$

Karla Rocha Liboreiro ${ }^{1}$ Renata Borges ${ }^{1}$

Universidade Federal de Minas Gerais ${ }^{1}$

Artigo recebido em 06.07.2016. Última versão recebida em 30.01.2017. Aprovado em 11.02.2017. Publicado online em 25.05.2017. 


\title{
Resumo
}

A adequação indivíduo-organização refere-se à congruência dos valores individuais do funcionário aos valores organizacionais. Essa adequação é explorada em diversos resultados individuais e organizacionais, tais como: desempenho, comprometimento, motivação, etc. Embora vários fatores tenham sido identificados como responsáveis por essa adequação, poucos estudos brasileiros exploraram quantitativamente o papel das redes sociais dentro das empresas. Esses relacionamentos construídos e mantidos dentro da organização são identificados pelos padrões de comunicação e interação entre os indivíduos, e podem interferir no nível de adequação indivíduoorganização. O objetivo desta pesquisa é compreender como as características das redes sociais, tais como grau de relacionamento, proximidade entre os atores e nível de intermediação, podem influenciar o nível de adequação indivíduo-organização. Realizou-se um survey com 116 funcionários de micro e pequenas empresas do setor de serviços. Os resultados da análise Partial Least Squares (PLS) indicam que o grau de relacionamento entre os funcionários influencia o nível de adequação indivíduo-organização. Ou seja, quanto mais o funcionário interage com seus colegas de trabalho, maior a probabilidade de adequação dos valores do indivíduo com os da organização.

Palavras-chave: redes sociais; adequação indivíduo-organização; gestão de pessoas.

\begin{abstract}
The person-organization fit (P-O Fit) refers to the congruence between individual and organizational values. This fit has been researched due to its importance for individual and organizational outcomes, such as: performance, commitment, motivation, and etc. Although several factors have been identified as predictor of this adaptation, few Brazilian studies have quantitatively explored the role of social networks within companies. These relationships which are built and kept inside the organization are identified by the patterns of communication and interaction among individuals, and they can influence the level of individual-organization fit. The objective of this research is to understand how the characteristics of social networks, such as relationship degree, closeness between actors, and intermediation, can influence the level of individual-organization fit. We surveyed 116 employees of micro and small companies in the service sector. Partial Least Squares (PLS) analysis results indicate that the degree of relationship between employees influences the level of P-O Fit. In other words, the more employees interact with each other, the greater the probability of fit between individual and organizational values.
\end{abstract}

Key words: social network; person-organization fit; human resource management. 


\section{Introdução}

A adequação indivíduo-organização é definida como o nível de congruência entre valores, competências cognitivas e habilidades do trabalhador e valores, demandas e características da organização em que ele trabalha (Youngs, Pogodzinski, Grogan, \& Perrone, 2015). Essa adequação é importante por ser sistematicamente associada a resultados individuais e organizacionais, tais como desempenho, rotatividade, satisfação no trabalho, comprometimento, etc. (Kiazad, Holtom, Hom, \& Newman, 2015; Kristof-Brown, Zimmerman, \& Johnson, 2005; Moynihan \& Pandey, 2007; Verquer, Beehr, \& Wagner, 2003; Zou, Ingram, \& Higgins, 2015). Os indivíduos são atraídos e permanecem em uma organização não somente por motivos financeiros, mas também pela oportunidade de realizar atividades que preenchem valores intrínsecos ao trabalhado. À medida que esses valores são satisfeitos, estabelece-se uma ligação psicológica entre as partes, gerando resultados positivos tanto de desempenho quanto de comprometimento, havendo, portanto, adequação entre indivíduo e organização.

Por outro lado, o sentimento de insatisfação com o trabalho pode ser gerado quando o trabalhador percebe que os valores da empresa em que ele trabalha não correspondem aos seus, e, consequentemente, suas expectativas em relação ao trabalho não são preenchidas. Esse sentimento pode levar a intenção do funcionário de deixar a empresa em busca de novas oportunidades (Edwards \& Cable, 2009). De fato, Wheeler, Coleman Gallagher, Brouer e Sablynski (2007) encontraram que os trabalhadores que sofrem com a falta de adequação indivíduo-organização apresentam baixos níveis de satisfação com o trabalho. No entanto, os achados de Wheeler et al. (2007) revelam ainda que o trabalhador insatisfeito deixa a organização somente quando percebe que existem alternativas ou oportunidades no mercado de trabalho.

As interações sociais ajudam os trabalhadores a desenvolver redes sociais no trabalho, levando a um sentimento de aceitação. Por outro lado, a falta de interação social resulta na percepção de baixa adequação com a organização (Cable \& Parson, 2001). As interações sociais ou redes sociais dentro das organizações são construções sociais, e, por isso, são alteradas e reproduzidas por meio das ações contínuas de seus membros (Nohria, 1992; Oliveira \& Lopes, 2014). Nesse sentido, as redes sociais atingem diretamente os trabalhadores que as integram, e, consequentemente, o modo que as empresas operam (Nelson, 2001; Nohria, 1992), tanto na resolução de conflitos (Nelson, 1989) e na transmissão de conhecimento (Argote, McEvily, \& Reagans, 2003) quanto na criação de oportunidades estratégicas (Tsai, 2001). A análise das redes sociais procura identificar as ligações diretas ou indiretas em um grupo delimitado de indivíduos, além de analisar as implicações desses vínculos (Steiner, 2006; Wasserman $\&$ Faust, 1994).

Nesta pesquisa, pretende-se compreender como as características das redes sociais formadas dentro das organizações podem interferir na maneira como o trabalhador percebe que seus valores são congruentes com os valores da empresa. Entende-se como características das redes sociais o grau de relacionamento dos trabalhadores, a proximidade entre os funcionários e o nível de intermediação entre eles. Portanto, o objetivo deste estudo é compreender como as características das redes sociais construídas dentro das empresas podem influenciar o nível de adequação indivíduo-organização.

Os resultados aqui podem oferecer uma compreensão da dinâmica das redes sociais constituídas dentro das organizações, relacionando-as com as percepções individuais sobre a adequação indivíduoorganização. Dessa maneira, pretende-se oferecer aos gestores e empresários participantes da pesquisa resultados empíricos para que possam desenvolver estratégias e ferramentas destinadas a melhorar a adequação entre indivíduo e empresa, e, consequentemente, a qualidade dos serviços prestados ao cliente.

Além de contribuir para a prática dos profissionais do setor de serviços, esta pesquisa oferece também implicações teóricas. A maioria dos estudos sobre redes sociais em administração no Brasil explora as redes estabelecidas entre as organizações, redes interorganizacionais (Ribeiro \& Porto, 2015), ou apresentam, como unidade de análise, setores específicos dentro da empresa (Ivar-Souza, De Muylder, \& Moriguchi, 2014). Outros estudos remetem também às redes sociais virtuais, como Facebook, Instagram, Whatsapp e etc. (Tubenchlak, Faveri, Zanini, \& Goldszmidt, 2015). Muitos deles 
analisam o comportamento do consumidor nessas redes sociais virtuais (Rocha, Ponchio, \& Rezende, 2015). Poucos são os estudos que focam no indivíduo e em suas relações sociais no ambiente organizacional apresentando a abordagem das redes (Fernandes, Santos, \& Fernandes, 2014), como, por exemplo, o trabalho de Murdocco e Oliva (2013).

Finalmente, esta pesquisa também contribui para a teoria ao relacionar as redes sociais construídas no ambiente de trabalho com o contexto organizacional. Dessa forma, os resultados deste trabalho atendem às sugestões de Mizruchi (2006) ao reconhecerem a importância da cultura nos trabalhos sobre redes sociais, fazendo com que o "bolo continue crescendo" (p. 82). Contudo, embora White (1993) tenha explorado o termo cultura da rede social de forma aprofundada, no sentido de identificar sistemas de significados e padrões de comportamentos, reconhece-se que este trabalho é um esforço inicial de compreender como a estrutura e as características das redes sociais interferem na adaptação do indivíduo no ambiente de trabalho.

\section{Referencial Teórico}

A adequação indivíduo-organização (P-O Fit) é conceituada como a compatibilidade entre os valores pessoais e organizacionais (Kristof, 1996; Youngs et al., 2015). Ao ingressar em uma organização, os valores e as crenças do indivíduo podem ou não se assemelhar aos valores da organização. As pessoas, quando se identificam e têm seus valores reconhecidos na prática organizacional, sentem-se mais motivadas e bem dispostas a realizar seus trabalhos (Latham \& Pinder, 2005). O P-O Fit é também considerado um dos fatores-chave para manter a competitividade da empresa, pois está relacionado diretamente aos níveis de comprometimento e motivação dos trabalhadores, gerando melhores resultados (Gregory, Albritton, \& Osmonbekov, 2010; Sekiguchi, 2007; Youngs et al., 2015).

Além de promover resultados individuais para a organização (Edwards \& Cable, 2009), o ajuste entre os valores pessoais e organizacionais tem sido relacionado a comportamentos que exigem maior envolvimento em atividades com benefícios coletivos. Esses benefícios referem-se principalmente ao reconhecimento da importância do indivíduo na organização, na distribuição de responsabilidades e na redução de conflitos (Elfenbein \& O'Reilly, 2007). Vianen, Shen e Chuang (2011), por exemplo, encontraram que a adequação dos valores individuais e organizacionais dos supervisores chineses promove não somente o comprometimento, como também melhora a qualidade das relações interpessoais. Isso ocorre porque, dentro das organizações, a qualidade da relação entre os funcionários e entre os funcionários e seus superiores é sensível aos níveis de confiança, à fluidez da comunicação e à previsibilidade dos comportamentos. Da mesma forma, os resultados de Tomei e Russo (2014) sugerem que o alinhamento cultural auxilia o trabalhador no engajamento de ações coletivas e não apenas nas ações de interesse próprio.

Outros estudos chamam a atenção para a importância do conteúdo das táticas de socialização como uma forma eficaz de promover a adequação dos valores individuais e organizacionais (Cable \& Parson, 2001; O'Reilly, Chatman, \& Caldwell, 1991). A socialização é compreendida como uma troca que ocorre por meio de atividades conjuntas, tais como conviver em um mesmo ambiente, manter conversas interativas e compartilhar conhecimento e experiências (Chang, Huang, Chiang, Hsu, \& Chang, 2012). Em geral, o trabalhador, quando ingressa em uma organização, passa a ter contato com outros funcionários e começa a conhecer a maneira na qual a organização funciona, que varia de práticas rotineiras até a tomada de decisão em situações de exceção (Chatman, 1991). O processo de socialização dos novos funcionários, de acordo com Bauer e Green (1998), assegura, dessa forma, a perpetuação dos valores e das normas da organização.

A socialização, quando bem realizada, pode proporcionar maior internalização dos valores organizacionais. Assim, os novos funcionários passam a se sentir parte da organização mais rapidamente e, com isso, apresentam atitudes e comportamentos desejáveis à organização (Bauer, Bodner, Erdogan, 
Truxillo, \& Tucker, 2007). As interações sociais planejadas permitem que o trabalhador recémcontratado reconheça seu papel na organização, resultando em comportamentos proativos (Perrot, Bauer, \& Roussel, 2012). Além disso, com o ajustamento, os funcionários ficam mais motivados e comprometidos (Youngs et al., 2015).

Durante o processo de socialização, a organização pode também influenciar os valores, atitudes e comportamentos dos indivíduos para facilitar seu ajuste aos valores da organização (Chatman, 1991). É nesse processo que acontece o primeiro contato com o ambiente organizacional, que, com o passar do tempo, dá aos novos trabalhadores o sentido da cultura da organização (Bauer \& Green, 1998). Em outras palavras, é por meio da interação social que ocorre a compreensão dos sistemas complexos e interdependentes das organizações (Evans, Wensley, \& Frissen, 2015; Wenger, McDermott, \& Snyder, 2002).

\section{Redes sociais e adequação indivíduo-organização}

Uma rede social, de acordo com Barnes (1972), compreende vínculos entre todos os membros da sociedade, ou entre parte deles, unidos por um propósito comum. Portanto, os indivíduos que integram uma rede social apresentam maior contato com os demais membros, fortalecem a confiança e geram um ambiente de maior compreensão e troca. Assim, a abordagem de redes consiste em uma alternativa para a análise da morfologia social, visto que a dinâmica das redes sociais altera algumas experiências coletivas. Além disso, esta abordagem representa também um novo caminho para auxiliar a compreensão de aspectos complexos, como fontes de dominação e transformação social, processos produtivos, e comportamentos interorganizacionais (Castells, 1999).

A perspectiva teórica das redes sociais é ainda utilizada nos estudos organizacionais como um recurso metodológico importante para analisar a relação entre os atores organizacionais, a partir dos sistemas de significados construídos entre eles (Bastos \& Santos, 2007). Partindo do pressuposto que as organizações são formadas por indivíduos que estabelecem relações, os mais diversos estudos organizacionais têm utilizado o método de análise de redes sociais para compreender melhor esses relacionamentos (Santos, Rossoni, \& Machado-da-Silva, 2011). O conceito de redes sociais abrange principalmente as redes informais de relações nas organizações. Uma importante pesquisa para o campo da análise de redes sociais foi a de Mayo (1990), na Hawthorne Electrical em Chicago, na qual foram investigados grupos de trabalhos nas fábricas e pequenas comunidades americanas. Esta pesquisa fortaleceu a ideia de que grupos sociais são redes de relações e de troca entre indivíduos, presentes também dentro das empresas.

A análise de redes sociais descreve os padrões das relações que conectam os indivíduos, o que permite estabelecer padrões de comunicação e relacionamento entre os atores (Zenk, Stadtfeld, \& Windhager, 2010). De acordo com Moynihan e Pandey (2007), quanto mais próximo o relacionamento entre indivíduos, maior a possibilidade de atuação conjunta na criação de novas ideias, aprendizado contínuo e alcance dos objetivos organizacionais. Os relacionamentos próximos formados por intenso contato, ou seja, laços fortes entre pessoas próximas, exigem reciprocidade, confiança e envolvimento emocional (Ahuja, 2000; Granovetter, 1983).

Por outro lado, Nelson (1989) examinou a relação entre redes sociais de trabalho e conflitos, concluindo que o baixo conflito caracteriza-se pelo grande número de laços fortes entre as várias redes sociais, medido como contato frequente. $\mathrm{O}$ autor enfatiza que a existência de contatos fortes dentro da organização pode também levar a aspectos negativos, como favoritismo ou até mesmo corrupção. Ao investigar as relações organizacionais e o comportamento antiético, Brass, Butterfield e Skaggs (1998) encontraram que pessoas com valores e atitudes similares tendem a desenvolver relacionamentos próximos dentro das empresas. Os autores acrescentam que, quando membros organizacionais concordam e partilham normas de conduta, padrões densos de relacionamentos emergem. Esses padrões podem ser positivos ou negativos, dependendo do sistema de valores dos indivíduos e, também, da organização. 
Contudo, a intensidade dos relacionamentos influencia ainda o nível de confiança entre os membros da rede social. Utilizando a abordagem de redes sociais, os achados de Lin (2007) mostram que o desenvolvimento da confiança entre os membros da organização cria um ambiente propício ao compartilhamento do conhecimento. Similarmente, Borges (2013) encontrou que relacionamentos fortes nas redes sociais das organizações desempenham um papel fundamental no processo de transferência de conhecimento tácito entre os profissionais de tecnologia da informação.

Em uma perspectiva macro, Rost (2011) examinou as redes entre os inventores da indústria automobilística alemã e colaboradores regulares. Os resultados indicam que os novos conhecimentos que foram obtidos fora da organização são divulgados de forma eficaz dentro do grupo, quando os membros estão densamente conectados. Kozan e Akdeniz (2014) acrescentam que, em empresas turcas, os relacionamentos próximos estão positivamente relacionados à expansão da produção e à aquisição do conhecimento. Finalmente, Kramarz e Skans (2014) constataram que jovens com pós-graduação se beneficiam de laços sociais fortes construídos também ao longo do curso, para ter acesso mais rápido aos postos de trabalho.

Ao analisar as redes sociais, Cross, Prusak e Parker (2002) afirmam que o conceito de centralidade é fundamental para compreender como os relacionamentos facilitam ou impedem o acesso aos recursos organizacionais. A medida de centralidade foi uma das primeiras medidas estudadas pelos analistas de redes sociais (Johnson, 2011). O conceito de centralidade difere da ideia de centralização. Scott (2012) explica que a centralidade ocorre quando um ponto é localmente central; assim, o trabalhador tem um grande número de conexões com outros em seu ambiente imediato. Já a centralização refere-se à coesão global ou à interação geral da rede, ou seja, propriedades particulares da estrutura da rede como um todo.

Para indicar o nível de centralidade do indivíduo na rede, as medidas de grau de contato, proximidade e intermediação são utilizadas. O grau de contato indica quão bem relacionado o indivíduo está dentro da rede, em relação à sua atividade de comunicação. A proximidade é definida por meio das distâncias de origem e destino que cada indivíduo possui em relação aos demais integrantes da rede. $\mathrm{Na}$ prática, a proximidade refere-se à facilidade que um indivíduo tem de recorrer a outro dentro da rede. Finalmente, a intermediação mede como o indivíduo conecta outros indivíduos na rede, ou seja, analisa se o indivíduo ocupa uma posição estratégica que permite o controle da comunicação.

Os indivíduos que apresentam elevado grau de contato na rede social, ou seja, relacionam-se intensamente com os demais membros da rede, ocupam posições mais centrais (Figueiredo, Chen, \& Azevedo, 2015). Essas pessoas estão em vantagem em relação aos membros mais periféricos, pois o elevado nível de relacionamento na rede permite que seus colegas de trabalho estejam familiarizados com suas ideias e cientes dos seus pontos de vista.

Além disso, funcionários com elevado grau de relacionamento no trabalho possuem acesso direto aos recursos e demonstram certa independência (Figueiredo et al., 2015). Segundo Perry-Smith e Mannucci (2015), a independência em relação aos colegas de trabalho está relacionada com a percepção de liberdade. Nesse sentido, Zou, Ingram, e Higgins (2015) acrescentam que uma das vantagens de se possuir um elevado grau de relacionamento na rede social intraorganizacional é conquistar a confiança e a autorização dos superiores para realizar o trabalho com maior autonomia.

A confiança recíproca e o apoio social entre pares também caracterizam o alto nível de relacionamento (Perry-Smith \& Mannucci, 2015). Os colegas de trabalho que se relacionam com mais frequência tendem a contar com ajuda extra para realizar atividades no trabalho, recebem apoio psicológico dos colegas em momentos de dificuldade e lidam melhor com o estresse no trabalho (Zou et al., 2015). Esses funcionários tendem a apresentar um melhor ajustamento em relação ao trabalho e à empresa porque possuem o sentimento de trabalhar em um ambiente familiar e apoiador.

Em outra perspectiva, as posições centrais nas redes sociais, em geral, são mais cobiçadas por representarem poder e controle. Os indivíduos que estão mais conectados na rede intraorganizacional desfrutam um status social elevado e apresentam expertise superior. Bolander, Satornino, Hughes, e 
Ferris (2015), por exemplo, encontraram que o grau de relacionamento afeta diretamente os resultados individuais. Os membros das equipes de vendas pesquisadas que ocupavam posições mais centrais, e que, portanto, desfrutavam de maior contato com seus colegas de trabalho, também apresentavam performance elevada.

Além do grau de relacionamento, o nível de proximidade também promove um senso de confiança dentro da empresa. As redes sociais com maior proximidade são caracterizadas pela presença de normas compartilhadas mais efetivas (Rost, 2011). Zou et al. (2015) acrescentam que as redes sociais intraorganizacionais cujos membros são próximos, cultivam um conjunto de expectativas coerentes, mantêm o controle da reputação de seus integrantes e garantem a manutenção da estabilidade e da segurança.

Para Kiazad, Holtom, Hom, e Newman (2015), com a proximidade dos colegas de trabalho, a influência social passa a ser incorporada na rotina da empresa. Os resultados da meta-análise realizada por Hülsheger, Anderson e Salgado (2009), mostram que a coesão dos colegas de trabalho está relacionada à criatividade das equipes de trabalho. Os autores explicam que, com a proximidade, criase um ambiente psicológico seguro, no qual os trabalhadores sentem-se confortáveis para explorar as situações de forma diferente, tendem a assumir riscos e estão mais dispostos a cooperar e a trocar ideias.

Funcionários que ocupam posições mais centrais nas redes intraorganizacionais também possuem acesso facilitado às informações e, portanto, maior controle sobre a comunicação. Essas pessoas apresentam elevado grau de intermediação, pois conectam diferentes pontos da rede, como departamentos, equipes e funcionários de vários níveis hierárquicos. Um gerente que reúne essas características tem acesso diferenciado ao conteúdo das informações que circulam pela rede se comparado a um estagiário, por exemplo, que tende a ocupar regiões periféricas da mesma rede. Bolander et al. (2015) afirmam que o acesso sobre o fluxo de informação na rede é particularmente relevante, pois garante não só o controle de acesso aos recursos como pode ser fonte de vantagem competitiva para empresa. $\mathrm{O}$ detentor ou centralizador da informação conta com um input diferenciado dentro da rede, apresentando soluções para problemas complexos que, muitas vezes, extrapolam as limitações dos demais membros. Essas pessoas desfrutam de status e prestígio dentro da rede social, e por isso, exercem influência sobre os outros integrantes da rede.

Portanto, os indivíduos que apresentam medidas de centralidade elevadas podem estar mais bem adaptados à organização. Moynihan e Pandey (2007), por exemplo, examinaram, sob a perspectiva de redes sociais, a relação entre adequação indivíduo, organização e intenção de deixar a empresa. Os autores concluem que os funcionários que apresentam elevada adequação ( $\mathrm{P}-\mathrm{O}$ fit) estão mais comprometidos em longo prazo com a organização. O'Reilly e Chatman (1986) explicam que a internalização de valores está positivamente relacionada a comportamentos pró-sociais. Sendo assim, funcionários com alta adequação de valores tendem a apresentar interações sociais e relacionamentos mais ativos, pois a ligação psicológica favorece a intenção de permancer na organização.

Neste sentido, espera-se que os funcionários que possuem um número maior de contato com diferentes colegas de trabalho dentro da organização, o façam porque estão bem adaptados à organização no que se refere ao ajustamento dos valores individuais com os valores organizacionais. Essas pessoas apresentam, portanto, um alto grau de relacionamento com os colegas de trabalho. Da mesma forma, o trabalhador que possui elevada adequação dos valores individuais e organizacionais tem facilidade para entrar em contato com seus colegas de trabalho, ou seja, tende a apresentar maior proximidade com os colegas de trabalho. Finalmente, espera-se que o funcionário que atua como intermediador com elevada frequência, ou seja, que atua como ponte de comunicação entre seus colegas de trabalho, o faça porque seus valores individuais coincidem com os valores organizacionais. Assim, as seguintes hipóteses são colocadas para pesquisa:

H1: O indivíduo que possui um alto grau de relacionamento com seus colegas de trabalho apresenta uma melhor adequação dos seus valores com os da organização. 
H2: O indivíduo que possui maior proximidade com seus colegas de trabalho apresenta uma melhor adequação dos seus valores com os da organização.

H3: O indivíduo que possui um alto grau de intermediação com seus colegas de trabalho apresenta uma melhor adequação dos seus valores com os da organização.

\section{Metodologia}

A pesquisa tem por objetivo verificar e analisar como as características dos laços sociais estabelecidos entre os funcionários das organizações pesquisadas influenciam a adequação indivíduoorganização. Para isso, seguindo o direcionamento de Babbie (2015) e Creswell (2013), realizou-se uma pesquisa explicativa, de natureza quantitativa, por meio de um survey.

A população pesquisada consiste em trabalhadores de empresas de pequeno porte do setor de serviços na região sudeste do país, mais especificamente no Estado de Minas Gerais. As empresas pesquisadas oferecem serviços nas áreas de engenharia, educacional, vendas, advocacia, contabilidade, construção civil, entre outras. Tais empresas são, em geral, escritórios caracterizados por funcionários com alto conhecimento técnico, que supostamente interagem de forma intensiva como membros de equipes formadas em torno de tarefas ou projetos específicos.

\section{Coleta de dados}

A coleta de dados foi realizada por meio da aplicação de questionários padronizados. Os respondentes foram selecionados aleatoriamente. No entanto, como a participação na pesquisa se deu de forma voluntária, pode-se caracterizar a amostra como intencional (Creswell, 2013). Seguiu-se ainda a recomendação de Babbie (2015) para que a amostra fosse selecionada considerando as implicações da homogeneidade e da representatividade nas organizações pesquisadas.

O questionário impresso foi administrado em todas as empresas pesquisadas da mesma forma. Primeiramente, o departamento de recursos humanos das empresas ofereceu uma listagem dos funcionários por departamento, para que os pesquisadores pudessem avaliar questões de representatividade. Os respondentes foram, então, selecionados randomicamente conforme as listagens fornecidas pelo departamento de recursos humanos das empresas. Depois, foi determinado pelas empresas, juntamente com os pesquisadores, dias e horários alternados para a aplicação dos questionários no próprio ambiente de trabalho e durante o expediente. Nesses dias, os pesquisadores explicaram para os respondentes que a participação na pesquisa era voluntária e aplicaram os questionários impressos. Foram obtidos 116 questionários válidos nesta pesquisa, suficiente para testar as hipóteses propostas, conforme indicou a análise de poder (power analysis).

O questionário é constituído de duas partes: a primeira, que investiga as questões relativas à intensidade dos laços entre os funcionários, e a segunda que mede o nível de adequação do indivíduoorganização. As questões que medem a frequência e a intensidade dos contatos entre os colegas de trabalho foram obtidas a partir dos trabalhos de Granovetter (1973), Lin (2007) e Nelson (1989). Ao utilizar esse instrumento, Borges (2013) encontrou um índice de confiabilidade de 0,94 (alfa de Cronbach) e variância extraída de $81 \%$. A segunda parte do questionário é composta por questões desenvolvidas por O'Reilly e Chatman (1986) para medir o nível de adequação dos valores individuais com os valores organizacionais. Pesquisas anteriores reportam alta confiabilidade e validade do instrumento com uma confiabilidade média de 0,76 (O’Reilly et al., 1991). Os itens do questionário estão descritos no Apêndice.

A escolha da escala social de respostas dos questionários permite que os respondentes avaliem e indiquem a intensidade com que concordam ou não com determinadas atitudes ou opiniões (Zikmund, Babbin, Carr, \& Griffin, 2009). Cada item do questionário está relacionado a uma escala social tipo 
Likert de cinco pontos, na qual o número um representa baixa aderência e se refere à expressão Discordo totalmente, variando até cinco, que representa alta aderência e se refere à expressão Concordo totalmente. Desse modo, cada item tem uma referência, que vai de 1 - Discordo totalmente, 2 Discordo, 3 - Nem discordo nem concordo, 4 - Concordo a 5 - Concordo totalmente.

Como a pesquisa social costuma tratar de construções subjetivas, que por natureza pode oferecer algum viés, a variância do método comum é frequentemente citada. Trata-se de uma variância sistemática introduzida nos dados como resultado da técnica de medição utilizada. Geralmente, são fontes da variância do método comum os questionários autoaplicados (porque as medidas são supostamente correlacionadas), a desejabilidade social (que pode inflar as correlações observadas), a afetividade negativa (pela tendência em discordar com os itens) e a aquiescência (com tendência em concordar com os itens). Como resultado, vários métodos estatísticos foram desenvolvidos para identificar esse problema (Malhotra, Kim, \& Patil, 2006).

O erro sistemático introduzido nos dados em decorrência da variância do método comum é representado pela diferença entre as relações observadas e as verdadeiras (Doty \& Glick, 1998). Spector (2006) e Malhotra, Kim e Patil (2006) argumentam que o possível erro introduzido decorrente do método de medição muitas vezes não é significativo e que, portanto, o pesquisador pode minimizá-lo antes de coletar os dados. Nesta pesquisa, procurou-se reduzir a probabilidade de inserir esse tipo de erro sistemático, seguindo as sugestões de Malhotra et al. (2006), ao contrabalançar a ordem das perguntas, utilizar instrumentos amplamente validados na literatura, aplicar escalas diferentes para medir os construtos, e, finalmente, assegurar a confidencialidade e o anonimato dos respondentes, informando que não existem respostas certas ou erradas, ou seja, que cada item deve ser respondido honestamente para representar a realidade.

\section{Análise dos dados}

A análise das redes sociais, ou seja, a frequência e a intensidade dos contatos, tais como centralidade, foram obtidas a partir do Software UCINET 6.268 para Windows, desenvolvido por Borgatti, Everett e Freeman (2002). Os resultados das análises do grau de relacionamento, proximidade com os nós da rede e intermediação entre os indivíduos foram exportados para serem utilizados como entrada de dados nas análises que testam as hipóteses desta pesquisa.

Finalmente, a análise das hipóteses de pesquisa foi realizada utilizando-se o método estatístico Partial Least Squares (PLS). Esse método foi escolhido porque é o mais apropriado para estimar o relacionamento entre variáveis quando a amostra é relativamente pequena (Chin, 1989). Além disso, o método PLS não requer o pressuposto de normalidade (Lohmöller, 1984). Primeiro analisou-se o modelo de mensuração para a amostra obtida, ou seja, quão bem os itens do questionário medem o que se propõem. O método de reamostragem bootstrap também foi empregado para determinar a significância dos caminhos (paths) dentro do modelo proposto.

\section{Resultados}

Esta pesquisa contou com 116 participantes, dentre os quais 52\% são mulheres e $48 \%$ são homens. A idade média dos respondentes é de 31 anos, com um desvio padrão de 9,46. A maioria da amostra é composta por indivíduos solteiros (55\%) que não possuem filhos, e 34\% são casados. A maioria dos participantes da pesquisa apresenta nível de escolaridade até o ensino médio (57\%), sendo que 30\% concluíram o ensino superior e 13\% fizeram algum tipo de especialização. Em relação ao cargo, $46 \%$ da amostra ocupa cargos técnicos ou auxiliares, 33\% são profissionais analistas, engenheiros, advogados, e etc., $12 \%$ ocupam cargos de liderança (coordenação ou gerência), e 10\% são diretores ou sócios da empresa. Finalmente, $67 \%$ dos respondentes afirmaram que são contratados das empresas participantes da pesquisa no regime de trabalho CLT, $5 \%$ são terceirizados, $8 \%$ são estagiários e $20 \%$ dos participantes optaram por não responder essa questão. 
Para avaliar a consistência interna do modelo de mensuração, foram analisados os valores das cargas, bem como a variância explicada e os valores de alfa de Cronbach para cada variável. A variável latente proximidade do nó (closeness) é obtida por meio das medidas de entrada e saída dos nós de cada membro da rede, ou seja, por meio das distâncias de origem e destino de cada nó. O resultado do modelo de mensuração indica que a medida da distância de saída de um nó para o outro não é significante para medir a proximidade do nó $(\lambda=-0,313, \mathrm{t}(112)=0,672, p=0,502)$. De fato, alguns autores afirmam que a distância de origem de cada nó (medida de entrada) é uma medida mais relevante de centralidade quando comparada à medida de saída, porque, em geral, o membro da rede tem menos controle sobre as conexões de entrada. Por isso, optou-se por retirar do modelo de mensuração a medida de saída (proximidade_OUT) da variável proximidade do nó (closeness). A Tabela 1 mostra os resultados do modelo de mensuração final.

Tabela 1

Cargas Fatoriais Padronizadas e Confiabilidade do Modelo de Mensuração

\begin{tabular}{lccccc}
\hline Construto & Indicadores & Cargas fatoriais & t-value* & $\begin{array}{c}\text { Variância } \\
\text { explicada }\end{array}$ & $\alpha$ de Cronbach \\
\hline FIT & FIT_1 & 0,78 & 12,59 & 0,63 & 0,85 \\
& FIT_2 & 0,74 & 10,20 & & \\
& FIT_3 & 0,88 & 32,00 & & \\
& FIT_4 & 0,82 & 17,91 & & \\
Grau de relacionamento & FIT_5 & 0,73 & 8,91 & & 0,94 \\
& Grau_IN & 0,98 & 125,70 & 0,95 & \\
Proximidade & Grau_OUT & 0,97 & 37,02 & & 1,00 \\
Intermediação & Proximidade_IN & 1,00 & & 1,00 & 1,00 \\
\hline
\end{tabular}

Nota. Fonte: Dados da pesquisa.

$* \mathrm{p}<0,001$.

Os demais construtos, com suas respectivas variáveis, obtiveram cargas fatoriais (factor loadings) superiores a $\pm 0,50$, valor considerado como o mínimo necessário para a obtenção da significância prática (Hair, Black, Babin, Anderson, \& Tatham, 2006). A validade convergente também superou o mínimo exigido de 0,50 ou $50 \%$, mostrando que os indicadores de cada variável dividem uma alta proporção de variância entre si. Finalmente, os valores de alfa de Cronbach para os construtos compostos por mais de uma variável apresentam alta consistência interna, com valores superiores a 0,85.

O grau de relacionamento entre os membros da rede é composto pelo número de laços que um nó (membro da rede) possui. Esses laços podem ser de entrada, ou seja, o número de laços direcionados para o nó, ou de saída, caracterizado pelo número de ligações direcionadas a outros nós. Além disso, os laços também dependem da medida de intermediação entre os membros da rede. A intermediação é uma medida do número de vezes que um nó atua como ponte para o menor caminho entre outros dois nós.

Já a medida do P-O Fit - adequação indivíduo-organização - foi obtida por meio de cinco questões: FIT_1 - Se os valores desta empresa fossem diferentes, eu não estaria tão vinculado a ela $(\mathrm{M}=3,51$, D.P. $=0,99)$; FIT_ 2 - Desde que eu entrei nesta empresa, considero que os meus valores pessoais e os da empresa são semelhantes $(M=3,60$, D.P. $=0,99)$; FIT_3 - Eu gosto de trabalhar nesta empresa por causa dos valores que ela defende e representa $(M=3,72$, D.P. $=0,92)$; FIT_4 Minha ligação com esta empresa está apoiada firmemente na afinidade dos meus valores com os da empresa $(M=3,72$, D.P. $=0,86)$; FIT_5 - Os valores que a empresa defende e representa são importantes para mim $(M=4,11$, D.P. $=0,82)$. O resultado geral indica que existe uma grande 
adequação dos valores individuais com os valores organizacionais $(\mathrm{M}=3,73$, D.P. $=0,73)$, pois a média obtida representa quase $75 \%$ do valor que indica a total adequação.

A Figura 1 mostra os resultados do PLS com os coeficientes de caminho (path) padronizados e o desvio padrão entre parêntesis. Como resultado, $\mathrm{H} 1$ foi confirmada, $\lambda=0,306, \mathrm{t}(112)=2,99, p<0,01$, indicando que o grau de relacionamento do funcionário na rede social da organização influencia positivamente a adequação dos seus valores individuais com os valores organizacionais (adequação indivíduo-organização). No entanto, as hipóteses H2 e H3 não foram confirmadas, sugerindo que o nível de adequação dos valores individuais com os valores organizacionais não depende da proximidade dos funcionários (proximidade dos nós), $\lambda=0,041, \mathrm{t}(112)=0,35, p=0,73$, e nem do grau de intermediação entre os indivíduos, $\lambda=0,065, \mathrm{t}(112)=0,42, p=0,67$.

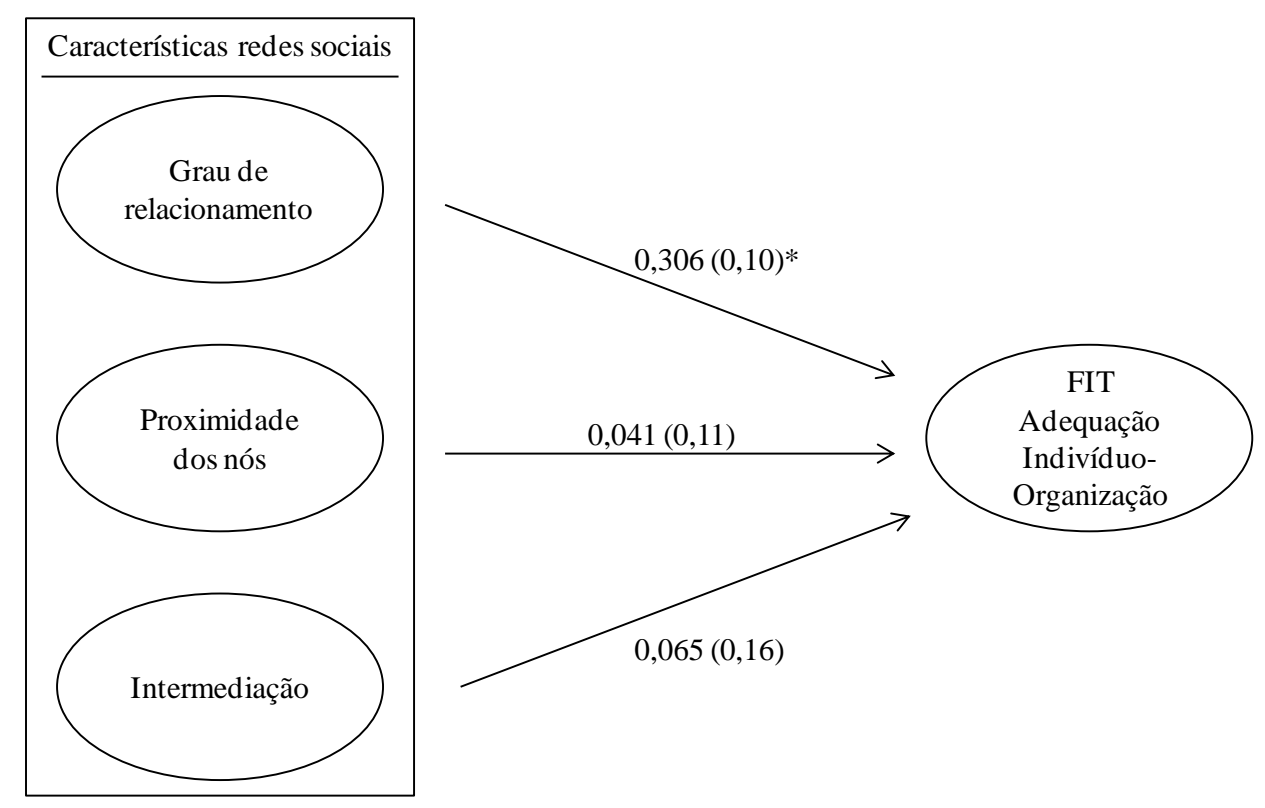

Figura 1. Resultado do Teste de Hipóteses $\mathrm{N}=116,{ }^{*} \mathrm{p}<0,01$.

Além dos coeficientes estimados com a análise do caminho (path analysis), a variância explicada da variável dependente adequação indivíduo-organização, tomando como base as variáveis independentes - grau de relacionamento, proximidade dos nós e intermediação - é um indicativo de quão bem os dados obtidos evidenciam ou dão suporte ao modelo proposto. Nesse caso, o resultado sugere que $11,5 \%$ da variação da adequação individuo-organização depende unicamente do grau de relacionamento na rede social formada dentro da organização $\left(\mathrm{R}^{2}=0,115, p<0,05\right)$.

\section{Implicações e Conclusões}

Poucas pesquisas em administração no Brasil têm abordado explicitamente o papel das redes sociais intraorganizacionais na adequação do indivíduo-organização. Nesse sentido, este trabalho tem como uma de suas principais contribuições a compreensão da influência dos relacionamentos sociais intraorganizacionais na congruência indivíduo-organização em empresas do setor terciário no Estado de Minas Gerais. Foi utilizada como medida de análise de redes sociais, a centralidade que possibilitou mensurar a relação entre o papel do indivíduo na rede e seu ajuste aos valores, demandas e características da organização.

As características das redes sociais relacionadas à medida de centralidade, como o grau de contato, a proximidade e a intermediação podem evidenciar padrões de cooperação, troca de informação, 
conhecimento e cultura entre os trabalhadores (Moynihan \& Pandey, 2007; Walker, 2013). Permitem também avaliar os resultados das táticas de socialização utilizadas para integrar o novo funcionário, posto que a análise das redes sociais revela relacionamentos formais e informais existentes no ambiente organizacional (Johnson, 2011).

Os resultados fornecem evidências significativas sobre os relacionamentos sociais e o ajustamento do trabalhador aos valores da organização. Assim como Brass et al. (1998) encontraram uma relação entre a proximidade dos relacionamentos e o desenvolvimento de valores e atitudes similares, as empresas do setor de serviço mineiro indicam que o grau de contato entre os indivíduos proporciona maiores níveis de adequação entre indivíduo-organização. Esse achado é justificado pela oportunidade de troca, cooperação, uniformização da comunicação e relacionamentos entre os trabalhadores (Zenk et al., 2010).

No entanto, as relações de proximidade e intermediação dos relacionamentos não foram confirmadas como fatores que influenciam a adequação do trabalhador com a organização. A ausência de confirmação da proximidade como relevante para a adequação do indivíduo-organização pode ser explicada pelo fato da maioria das empresas do setor de serviço ser micro ou pequena empresa, em que os trabalhadores possuem grande proximidade física, facilitando o contato entre eles. O mesmo pode ser afirmado sobre os relacionamentos de intermediação entre os trabalhadores. Nelson (1989) aponta que os relacionamentos em grupo são fontes de inovação, cooperação e diminuição de conflitos. $\mathrm{O}$ relacionamento entre os funcionários contribui para consenso dos interesses e aumento do desempenho organizacional. Contudo, as empresas de serviços estudadas possuem número reduzido de trabalhadores, o que diminui a incidência de relacionamentos mediadores e, consequentemente, a necessidade de intermediação.

Esta pesquisa pretende contribuir para a teoria de redes sociais com o estudo das micro e pequenas empresas do setor de serviços mineiro. Os resultados evidenciam a importância da compreensão dos relacionamentos intraorganizacionais para promover maiores níveis de adequação entre os valores individuais e organizacionais. Em especial, o setor de serviços exige um envolvimento direto dos funcionários para aumentar a qualidade dos serviços e a satisfação dos clientes. Assim, ao evidenciar que o grau de relacionamento entre os funcionários interfere no nível de adequação indivíduoorganização, este estudo busca ampliar a percepção da relevância das táticas de socialização na construção de um planejamento de integração de novos trabalhadores.

Portanto, compreender a dinâmica dos relacionamentos entre os funcionários proporciona ao gestor a oportunidade de delinear ações efetivas para fomentar comportamentos de cooperação mútua, construção coletiva, ajustes aos valores e às demandas da organização. A análise da rede social intraorganizacional resulta em um aprofundamento nas características das interações, compartilhamento de aprendizagens e experiências ocorridas nas atividades conjuntas. Torna-se importante para a gestão compreender a distribuição da rede social intraorganizacional e o papel de cada funcionário dentro do ambiente organizacional, promovendo o contato entre os membros da rede para melhorar o nível de adequação dos funcionários com a empresa.

Contudo, o tamanho restrito da amostra pode ser uma fonte de limitação aos achados, pois as empresas de serviços pesquisadas possuem equipes pequenas e altamente especializadas, o que impossibilita generalizações. E também, a população apresenta grande diversidade de segmentos, pois as empresas estudadas abarcam serviços tais como educação, engenharia, contabilidade, consultoria e outros. Outra limitação relevante ao trabalho é a investigação da percepção dos trabalhadores quanto aos relacionamentos e a adequação à organização. No entanto, entender a percepção do papel do indivíduo dentro da rede contribui para compreensão da dinâmica dos relacionamentos e táticas de socialização a partir da subjetividade do trabalhador, e amplia a percepção do papel exercido por ele.

Questões relativas à endogeneidade são também fontes de limitação para este estudo. A endogeneidade ocorre quando a variável independente está correlacionada com o erro, comprometendo os resultados estatísticos (Antonakis, Bendahan, Jacquart, \& Lalive, 2014). Problemas de endogeneidade podem acontecer devido à omissão de variáveis, simultaneidade e explicações rivais. 
Em relação à omissão de variáveis, reconhece-se que os níveis de adequação indivíduo-organização podem ser explicados por outras variáveis independentes A adequação ou inadequação entre os valores individuais e organizacionais podem decorrer de diversos outros fatores, como, por exemplo, processos seletivos ineficazes que geram expectativas que não correspondem à realidade da empresa, ou da própria estrutura de poder, caracterizada muitas vezes pelo estilo de liderança de seus membros. Sugere-se, portanto, que pesquisas futuras explorem outros antecedentes na perspectiva das redes sociais.

Problemas de simultaneidade podem acontecer quando ambas as variáveis estudadas se influenciam, ou seja, o grau de relacionamento, proximidade e intermediação afetam e, ao mesmo tempo, são afetados pelo nível de adequação indivíduo-organização. Para testar essa hipótese, é necessário desenvolver um estudo longitudinal ou dinâmico, ou desenvolver ainda um experimento (Antonakis et al., 2014; Melcher \& Melcher, 1980). Embora as hipóteses colocadas nesta pesquisa contenham com o suporte teórico e empírico, reconhece-se que pesquisas sobre redes sociais intraorganizacionais se posicionem na fase exploratória/explicativa, ou seja, ainda não existe um entendimento consolidado na literatura sobre causas e consequências relacionais.

Finalmente, explicações rivais podem existir também quando ambas as variáveis, independente e dependente, são influenciadas por uma terceira variável não observada e, portanto, não inserida na equação proposta. É principalmente nesse sentido que Antonakis, Bendahan, Jacquart e Lalive (2014) explicam a endogeneidade. Assim, seria necessário desenvolver uma pesquisa para compreender se questões de centralidade nas redes sociais (como grau de relacionamento, proximidade e intermediação) são afetadas pelas mesmas questões que potencialmente podem afetar o nível de adequação indivíduoorganização.

Além disso, outra limitação decorre da coleta de dados, pois existem outras opções de instrumentos de medição disponíveis na literatura que podem ser testados para a realidade brasileira. Finalmente, ainda sobre a coleta de dados, entrevistas podem ser realizadas em um segundo momento para permitir a triangulação dos dados e, com isso, aumentar a confiabilidade dos achados.

Para pesquisas futuras, sugere-se o estudo de empresas em um mesmo segmento de prestação de serviço, o que possibilitará avaliações das táticas de socialização e da dinâmica dos relacionamentos. A investigação da percepção dos trabalhadores em relação ao relacionamento intraorganizacional pode ser realizada também com o auxílio de entrevistas e observações não participantes nas empresas. E, por fim, pode-se ainda contemplar as díades e tríades formadas dentro da rede das empresas para compreender como a dinâmica de cada área e sua relação com as demais áreas influenciam na adequação indivíduoorganização.

As empresas do setor de serviços são responsáveis por grande parte das transações econômicas e da coprodução de inovações tecnológicas. Desse modo, a adequação do indivíduo-organização é relevante por produzir maior qualidade nos serviços e maior satisfação dos clientes. Nesta perspectiva, os relacionamentos intraorganizacionais são relevantes para atingir melhores níveis de ajuste do trabalhador à organização, uma vez que o grau de relacionamento entre os funcionários influencia o nível de adequação indivíduo-organização.

\section{Referências}

Ahuja, G. (2000). Collaboration networks, structural holes, and innovation: A longitudinal study. Administrative Science Quarterly, 45(3), 425-455. http://dx.doi.org/10.2307/2667105

Antonakis, J., Bendahan, S., Jacquart, P., \& Lalive, R. (2014). Causality and endogeneity: Problems and solutions. In D. V. Day (Ed.), The Oxford handbook of leadership and organizations (pp. 93117). New York: Oxford University Press. 
Argote, L., McEvily, B., \& Reagans, R. (2003). Managing knowledge in organizations: An integrative framework and review of emerging themes. Management Science, 49(4), 571-582. http://dx.doi.org/10.1287/mnsc.49.4.571.14424

Babbie, E. (2015). The practice of social research (14th ed.). Boston, MA: Cengage Learding.

Barnes, J. A. (1972). Social networks. An Addison-Wesley module in anthropology. Module 26. Reading, MA: Addison-Wesley Publishing Co.

Bastos, V. B., \& Santos, M. V. (2007). Redes sociais informais e compartilhamento de significados sobre mudança organizacional. Revista de Administração de Empresas, 47(3), 1-13. http://dx.doi.org/10.1590/S0034-75902007000300003

Bauer, T. N., Bodner, T., Erdogan, B., Truxillo, D. M., \& Tucker, J. S. (2007). Newcomer adjustment during organizational socialization: A meta-analytic review of antecedents, outcomes, and methods. Journal of Applied Psychology, 92(3), 707-721. http://dx.doi.org/10.1037/00219010.92.3.707

Bauer, T. N., \& Green, S. G. (1998). Testing the combined effects of newcomer information seeking and manager behavior on socialization. Journal of Applied Psychology, 83(1), 72-83. http://dx.doi.org/10.1037/0021-9010.83.1.72

Bolander, W., Satornino, C. B., Hughes, D. E., \& Ferris, G. R. (2015). Social networks within sales organizations: Their development and importance for salesperson performance. Journal of Marketing, 79(6), 1-16. http://dx.doi.org/10.1509/jm.14.0444

Borgatti, S. P., Everett, M. G., \& Freeman, L. C. (2002). UCINET 6 for Windows: Software for social network analysis. Harvard, MA: Analytic Technologies.

Borges, R. (2013). Tacit knowledge sharing between IT workers: The role of organizational culture, personality, and social environment. Management Research Review, 36(1), 89-108. http://dx.doi.org/10.1108/01409171311284602

Brass, D. J., Butterfield, K. D., \& Skaggs, B. C. (1998). Relationships and unethical behavior: A social network perspective. Academy of Management Review, 23(1), 14-31. http://dx.doi.org/10.5465/AMR.1998.192955

Cable, D. M., \& Parson, C. K. (2001). Socialization tactics and person-organization fit. Personnel Psychology, 54(1), 1-23. http://dx.doi.org/10.1111/j.1744-6570.2001.tb00083.x

Castells, M. (1999). A sociedade em Rede-A era da informação: economia, sociedade e cultura. São Paulo: Paz e Terra.

Chang, C.-W., Huang, H.-C., Chiang, C.-Y., Hsu, C.-P., \& Chang, C.-C. (2012). Social capital and knowledge sharing: Effects on patient safety. Journal of Advanced Nursing, 68(8), 1793-1803. http://dx.doi.org/10.1111/j.1365-2648.2011.05871.x

Chatman, J. A. (1991). Matching people and organizations: Selection and socialization in public accounting firms. Administrative Science Quarterly, 36(3), 459-484. http://dx.doi.org/10.2307/2393204

Chin, W. W. (1989). The partial least squares approach to structural equation modeling. In G. A. Marcoulides (Ed.), Modern methods for business research (pp. 295-358). Mahwah: Lawrence Erlbaum Associates.

Creswell, J. W. (2013). Research design: Qualitative, quantitative, and mixed methods approaches (4th ed.). Thousand Oaks, CA: Sage Publications. 
Cross, R., Prusak, L., \& Parker, A. (2002). Where work happens: The care and feeding of informal networks in organizations. Cambridge, MA: Institute for Knowledge-based Organizations.

Doty, D. H., \& Glick, W. H. (1998). Common methods bias: Does common methods variance really bias results? Organizational Research Methods, 1(4), 374-406. http://dx.doi.org/10.1177/109442819814002

Edwards, J. R., \& Cable, D. M. (2009). The value of value congruence. The Journal of Applied Psychology, 94(3), 654-677. http://dx.doi.org/10.1037/a0014891

Elfenbein, H. A, \& O'Reilly C. A. (2007). Fitting in: the effects of relational demography and personculture fit on group process and performance. Group and Organization Management, 32(1), 109142. https://doi.org/10.1177/1059601106286882

Evans, M., Wensley, A., \& Frissen, L. (2015). The mediating effects of trustworthiness on socialcognitive factors and knowledge sharing in a large professional service firm. The Electronic Journal of Knowledge Management, 13(3), 240-253.

Fernandes, B. K., Santos, L. G. A., \& Fernandes, B. H. R. (2014). Níveis de complexidade e inserção em uma rede social de comunidades terapêuticas. Revista de Administração Contemporânea, 18(4), 446-464. Recuperado de http://www.scielo.br/pdf/rac/v18n4/1415-6555-rac-18-0400446.pdf. http://dx.doi.org/10.1590/1982-7849rac20141193

Figueiredo, C., Chen, W., \& Azevedo, J. (2015). Central nodes and surprise in content selection in social networks. Computers in Human Behavior, 51, Part A, 382-392. http://dx.doi.org/10.1016/j.chb.2015.04.070

Granovetter, M. (1973). The strength of weak ties. American Journal of Sociology, 78(6), 1360-1380. Retrieved from http://www.jstor.org/stable/2776392. http://dx.doi.org/10.1086/225469

Granovetter, M. (1983). The strength of weak ties: A network theory revisited. Sociological Theory, 1, 201-233. http://dx.doi.org/10.2307/202051

Gregory, B. T., Albritton, M. D., \& Osmonbekov, T. (2010). The mediating role of psychological empowerment on the relationships between $\mathrm{P}-\mathrm{O}$ fit, job satisfaction, and in-role performance. Journal of Business and Psychology, 25(4), 639-647. http://dx.doi.org/10.1007/s10869-010-9156-7

Hair, J. F., Black, W. C., Babin, B. J., Anderson, R. E., \& Tatham, R. L. (2006). Multivariate data analysis (6th ed.). Upper Saddle River, NJ: Pearson Prentice Hall.

Hülsheger, U. R., Anderson, N., \& Salgado, J. F. (2009). Team-level predictors of innovation at work: A comprehensive meta-analysis spanning three decades of research. Journal of Applied Psychology, 94(5), 1128-1145. http://dx.doi.org/10.1037/a0015978

Ivar-Souza, F., De Muylder, C. F., \& Moriguchi, S. N. (2014). Redes sociais e os impactos dessa inovação nas organizações: Estudo de caso da rede corporativa "Comunidade de Negócios" da área comercial das empresas do Grupo Algar. Revista Gestão \& Tecnologia, 14(1), 225-244. http://dx.doi.org/10.20397/2177-6652/2014.v14i1.516

Johnson, D. J. (2011). Gestão de redes de conhecimento. São Paulo: SENAC.

Kiazad, K., Holtom, B. C., Hom, P. W., \& Newman, A. (2015). Job embeddedness: A multifoci theoretical extension. Journal of Applied Psychology, 100(3), 641-659. http://dx.doi.org/10.1037/a0038919

Kozan, M., \& Akdeniz, L. (2014). Role of strong versus weak networks in small business growth in an emerging economy. Administrative Sciences, 4(1), 35-50. http://dx.doi.org/10.3390/admsci4010035 
Kramarz, F., \& Skans, O. N. (2014). When strong ties are strong: Networks and youth labour market entry. The Review of Economic Studies, 81(3), 1164-1200. http://dx.doi.org/10.1093/restud/rdt049

Kristof, A. L. (1996). Person-organization fit: An integrative review of its conceptualizations, measurement, and implications. Personnel Psychology, 49(1), 1-49. http://dx.doi.org/10.1111/j.1744-6570.1996.tb01790.x

Kristof-Brown, A. L., Zimmerman, R. D., \& Johnson, E. C. (2005). Consequences of individual's fit at work: a meta-analysis of person job, person organisation, person group, and person supervisor fit. Personnel Psychology, 58(2), 281-342. http://dx.doi.org/10.1111/j.1744-6570.2005.00672.x

Latham, G. P., \& Pinder, C. C. (2005). Work motivation theory and research at the dawn of the twentyfirst century. Annual Review Psychology., 56, 485-516. http://dx.doi.org/10.1146/annurev.psych.55.090902.142105

Lin, C.-P. P. (2007). To share or not to share: Modeling tacit knowledge sharing, its mediators and antecedents. Journal of Business Ethics, 70(4), 411-428. http://dx.doi.org/10.1007/s10551-0069119-0

Lohmöller, J.-B. (1984). LVPLS program manual. Köln: Zentralarchiv für Empirische Sozialforschung.

Malhotra, N. K., Kim, S. S., \& Patil, A. (2006). Common method variance in IS research: a comparison of alternative approaches and a reanalysis of past research. Management Science, 52(12), 18651883. http://dx.doi.org/10.1287/mnsc.1060.0597

Mayo, E. (1990). Hawthorne and the Western Electric Company. In D. S. Pugh (Ed.), Organization theory: Selected reading (pp. 345-357). London: Penguin Press.

Melcher, A. J., \& Melcher, B. H. (1980). Toward a systems theory of policy analysis: Static versus dynamic analysis. Academy of Management Review, 5(2), 235-247. http://dx.doi.org/10.5465/AMR.1980.4288738

Mizruchi, M. S. (2006). Análise de redes sociais: Avanços recentes e controvérsias atuais. Revista de Administração de Empresas, 46(3), 72-86. http://dx.doi.org/10.1590/S003475902006000300013

Moynihan, D. P., \& Pandey, S. K. (2007). The ties that bind: Social networks, person-organization value fit, and turnover intention. Journal of Public Administration Research and Theory, 18(2), 205227. http://dx.doi.org/10.1093/jopart/mum013

Murdocco, S. D. O., \& Oliva, E. D. C. (2013). A cultura organizacional e a centralidade nas redes sociais: Um estudo exploratório em uma empresa de serviços. Revista de Negócios, 18(3), 58-75. http://dx.doi.org/10.7867/1980-4431.2013v18n3p58-75

Nelson, R. E. (1989). The strength of strong ties: Social networks and intergroup conflict in organizations. Academy of Management Journal, 32(2), 377-401. http://dx.doi.org/10.2307/256367

Nelson, R. E. (2001). On the shape of verbal networks in organizations. Organization Studies, 22(5), 797-823. http://dx.doi.org/10.1177/0170840601225003

Nohria, N. (1992). Introduction: Is a network perspective a useful way of studying organizations? In N. Nohria \& R. G. Eccles (Eds.), Networks and organizations: Structure, form, and action (pp. 544558). Boston: Harvard Business School Press.

Oliveira, C. C. G., \& Lopes, H. E. G. (2014). Coopetição em redes interpessoais. Revista de Administração Contemporânea, 18(4), 508-522. Recuperado de 
http://www.scielo.br/pdf/rac/v18n4/1415-6555-rac-18-04-00508.pdf. http://dx.doi.org/10.1590/1982-7849rac20141096

O'Reilly, C. A., \& Chatman, J. A. (1986). Organizational commitment and psychological attachment: The effects of compliance, identification, and internalization on prosocial behavior. Journal of Applied Psychology, 71(3), 492-499. http://dx.doi.org/10.1037//0021-9010.71.3.492

O'Reilly, C. A., Chatman, J., \& Caldwell, D. F. (1991). People and organizational culture: A profile comparison approach to assessing person-organization fit. Academy of Management Journal, 34(3), 487-516. http://dx.doi.org/10.2307/256404

Perrot, S., Bauer, T. N., \& Roussel, P. (2012). Organizational socialization tactics: Determining the relative impact of context, content, and social tactics. Revue de Gestion Des Ressources Humaines, 86(4), 21-35. http://dx.doi.org/10.3917/grhu.086.0021

Perry-Smith, J. E., \& Mannucci, P. V. (2015). Social networks, creativity, and entrepreneurship. In C. E. Shalley, M. A. Hitt, \& J. Zhou (Eds.), The Oxford handbook of creativity, innovation, and entrepreneurship (pp. 205-224). New York: Oxford University Press.

Ribeiro, W. L., \& Porto, G. S. (2015). Analysis of innovation networks financed by biotechnology and energy sector funds. Review of Business Management, 17(58), 1426-1443. http://dx.doi.org/10.7819/rbgn.v17i58.1764

Rocha, V. M., Ponchio, M. C., \& Rezende, F. (2015). Lealdade do consumidor e programas de fidelidade: Uma análise topográfica do campo de conhecimento à luz da bibliometria da estatística espacial das redes sociais. Revista de Ciências da Administração, 17(41), 9-20. http://dx.doi.org/10.5007/2175-8077.2015v17n41p9

Rost, K. (2011). The strength of strong ties in the creation of innovation. Research Policy, 40(4), 588604. http://dx.doi.org/10.1016/j.respol.2010.12.001

Santos, L. G. A. dos, Rossoni, L., \& Machado-da-Silva, C. L. (2011). Condicionantes estruturais dos relacionamentos intraorganizacionais: Uma análise da influência sobre relações de comunicação e decisão. Revista de Administração Mackenzie, 12(1), 139-168. http://dx.doi.org/10.1590/S1678-69712011000100006

Scott, J. (2012). Social network analysis. London: Sage.

Sekiguchi, T. (2007). A contingency perspective of the importance of PJ fit and PO fit in employee selection. Journal of Managerial Psychology, 22(2), 118-131. http://dx.doi.org/10.1108/02683940710726384

Spector, P. E. (2006). Method variance in organizational research truth or urban legend? Organizational Research Methods2, 9(2), 221-323. http://dx.doi.org/10.1177/1094428105284955

Steiner, P. (2006). A sociologia econômica. São Paulo: Atlas.

Tomei, P. A., \& Russo, G. M. (2014). Análise do alinhamento cultural de uma organização: Um estudo de caso da Arfco. Gestão \& Planejamento, 15(2), 382-409.

Tsai, W. (2001). Knowledge transfer in intraorganizational networks: Effects of network position and absorptive capacity on business unit innovation and performance. Academy of Management Journal, 44(5), 996-1004. http://dx.doi.org/10.2307/3069443

Tubenchlak, D. B., Faveri, D. de, Zanini, M. T., \& Goldszmidt, R. (2015). Motivações da comunicação boca a boca eletrônica positiva entre consumidores no Facebook. Revista de Administração Contemporânea, 19(1), 107-126. Recuperado de http://www.scielo.br/pdf/rac/v19n1/1982-7849rac-19-1-0107.pdf. http://dx.doi.org/10.1590/1982-7849rac20151998 
Vianen, A. E. M. van, Shen, C.-T., \& Chuang, A. (2011). Person-organization and person-supervisor fits: Employee commitments in a Chinese context. Journal of Organizational Behavior, 32(6), 906-926. http://dx.doi.org/10.1002/job.726

Verquer, M. L., Beehr, T. A., \& Wagner, S. H. (2003). A meta-analysis of relations between personorganization fit and work attitudes. Journal of Vocational Behavior, 63(3), 473-489. http://dx.doi.org/10.1016/S0001-8791(02)00036-2

Walker, W. J. (2013). The New Work contract: Mitigating the negative effects on work attitudes. Journal of Organizational Culture, Communication and Conflict, 17(2), 121-136.

Wasserman, S., \& Faust, K. (1994). Social network analysis: Methods and applications. Cambridge. UK: Cambridge University Press.

Wenger, E., McDermott, R., \& Snyder, W. M. (2002). Cultivating communities of practice. Cambridge, MA: Harvard Business School Press.

Wheeler, A. R., Coleman Gallagher, V., Brouer, R. L., \& Sablynski, C. J. (2007). When personorganization (mis)fit and (dis)satisfaction lead to turnover. Journal of Managerial Psychology, 22(2), 203-219. http://dx.doi.org/10.1108/02683940710726447

White, H. C. (1993). Careers and creativity: social forces in the art. Boulder, CO: Westview.

Youngs, P., Pogodzinski, B., Grogan, E., \& Perrone, F. (2015). Person-organization fit and research on instruction. Educational Researcher, 44(1), http://dx.doi.org/10.3102/0013189X15569531

Zenk, L., Stadtfeld, C., \& Windhager, F. (2010). How to analyze dynamic network patterns of high performing teams. Procedia - Social and Behavioral Sciences, 2(4), 6418-6422. http://dx.doi.org/10.1016/j.sbspro.2010.04.051

Zikmund, W. G., Babbin, B. J., Carr, J. C., \& Griffin, M. (2009). Business research methods (8th ed.). Cincinnati: South-Western College Pub.

Zou, X., Ingram, P., \& Higgins, E. T. (2015). Social networks and life satisfaction: The interplay of network density and regulatory focus. Motivation and Emotion, 39(5), 693-713. http://dx.doi.org/10.1007/s11031-015-9490-1

\section{Dados dos Autores}

Maria Gabriela de Cássia Miranda

Av. Antônio Carlos 6627, sala 4012, Pampulha, 31270-901, Belo Horizonte, MG, Brasil. E-mail: mgabriela.miranda@gmail.com; gabriela_ufop@yahoo.com.br

Karla Rocha Liboreiro

Av. Antônio Carlos 6627, sala 4012, Pampulha, 31270-901, Belo Horizonte, MG, Brasil. E-mail: karlarliboreiro@ gmail.com

Renata Borges

Av. Antônio Carlos 6627, sala 4012, Pampulha, 31270-901, Belo Horizonte, MG, Brasil. E-mail: renatasg@ face.ufmg.br; renata.guimaraes.borges@gmail.com 


\section{APÊNDICE}

\section{Itens do Questionário da Pesquisa}

\section{Redes Sociais - Redes de Relacionamento, Proximidade dos Nós e Intermediação}

A primeira coluna do questionário contém o nome de todas as pessoas do seu departamento e das demais áreas da empresa. Marque um X na coluna que melhor descreve a frequência do seu contato com cada pessoa.

\begin{tabular}{|c|c|c|c|c|c|}
\hline \multirow{2}{*}{$\begin{array}{l}\text { Nome dos } \\
\text { Funcionários } \\
\text { da Empresa }\end{array}$} & \multicolumn{5}{|c|}{ Frequência dos contatos } \\
\hline & $\begin{array}{l}\text { Nunca } \\
\text { (nenhuma vez } \\
\text { no mês) }\end{array}$ & $\begin{array}{l}\text { Raras vezes } \\
\text { (algumas vezes } \\
\text { no mês) }\end{array}$ & $\begin{array}{l}\text { Às vezes } \\
\text { (até } 1 \text { vez por } \\
\text { semana) }\end{array}$ & $\begin{array}{l}\text { Frequentemente } \\
\text { (de } 2 \text { a } 4 \text { vezes } \\
\text { por semana) }\end{array}$ & $\begin{array}{l}\text { Todos os dias } \\
\text { da semana }\end{array}$ \\
\hline \multicolumn{6}{|l|}{ Funcionário $\mathrm{A}$} \\
\hline \multicolumn{6}{|l|}{ Funcionário B } \\
\hline Funcionário $\mathrm{C}$ & & & & & \\
\hline
\end{tabular}

\section{FIT - Adequação Indivíduo-Organização}

1. Se os valores da empresa fossem diferentes, eu não estaria tão vinculado a ela.

2. Desde que eu entrei nesta empresa, considero que meus valores pessoais e os da empresa são semelhantes.

3. Eu gosto de trabalhar nesta empresa por causa dos valores que ela defende e representa.

4. Minha ligação com esta empresa está apoiada firmemente na afinidade dos meus valores com os da empresa.

5. O que a empresa defende e representa são importantes para mim. 